This item was submitted to Loughborough's Research Repository by the author.

Items in Figshare are protected by copyright, with all rights reserved, unless otherwise indicated.

\title{
Comparing additive manufacturing technologies for customised wrist splints
}

PLEASE CITE THE PUBLISHED VERSION

http://dx.doi.org/10.1108/RPJ-10-2013-0099

PUBLISHER

(c) Emerald Group Publishing Limited

VERSION

AM (Accepted Manuscript)

LICENCE

CC BY-NC-ND 4.0

REPOSITORY RECORD

Paterson, Abby, Richard Bibb, lan Campbell, and Guy Bingham. 2015. "Comparing Additive Manufacturing Technologies for Customised Wrist Splints”. Loughborough University. https://hdl.handle.net/2134/17656. 


\section{Comparison of Additive Manufacturing Systems for the Design and Fabrication of Customised Wrist Splints}

Purpose - The purpose of this paper is to compare four different additive manufacturing (AM) processes in order to assess their suitability in the context of upper extremity splinting.

Design/methodology/approach - This paper describes the design characteristics and subsequent fabrication of six different wrist splints using four different AM processes: Laser Sintering (LS), Fused Deposition Modelling (FDM), Stereolithography (SLA) and PolyJet material jetting via Objet Connex. The suitability of each process was then compared against competing designs and processes from traditional splinting. The splints were created using a digital design workflow that combined recognised clinical best practice with design for AM principles.

Findings - Research concluded that, based on currently available technology, FDM was considered the least suitable AM process for upper extremity splinting. LS, SLA and material jetting show promise for future applications but further research and development into AM processes, materials and splint design optimisation are required if the full potential is to be realised.

Originality/value - Unlike previous work that has applied AM processes to replicating traditional splint designs, the splints described are based on a digital design for AM workflow, incorporating novel features and physical properties not previously possible in clinical splinting. The benefits of AM for customised splint fabrication have been summarised. A range of AM processes have also been evaluated for splinting, exposing the limitations of existing technology, demonstrating novel and advantageous design features and opportunities for future research.

Keywords Additive Manufacture, Heterogeneous, Orthotic, Textile, Wrist Splint.

\section{Introduction}

Wrist splints provide multi-faceted treatment outcomes to patients, including pain relief through immobilisation of affected joints (Callinan and Mathiowetz, 1996; Jacobs, 2003). Wrist immobilisation splints, for example, are designed to immobilise the wrist whilst allowing mobility of all digits to promote endurance to everyday tasks (Pagnotta et al., 2005).

There are two main categories of splints; prefabricated 'off-the-shelf' splints and custom-made splints. Prefabricated splints can be bought from a variety of stores such as pharmacies, but may also be prescribed by splinting practitioners, such as occupational therapists or physiotherapists. Prefabricated splints may come in a range of sizes (e.g. small, medium and large) which assumes a 'one size fits all' strategy, and not necessarily tailored to suit an individual unless adjusted by the user or a splinting practitioner. Alternatively, custom-made splints are produced and distributed exclusively by splinting practitioners to suit each individual patient's lifestyle, as well as anatomical demands relative to their condition. Custom-made splints offer superior fit and comfort and in many circumstances can be less bulky than off-the-shelf items. They can also be made to accommodate extremes of size and deformity that is not always possible with off-the-shelf items, which inevitably have limits on their adjustability. Custom-made splints maintain their shape at all times whilst off-the-shelf items need to be adjusted each time they are put on and it is not always possible to replicate the adjustment precisely on each occasion. Consequently off-the-shelf splints cannot accommodate every patient and there will always be a need for custom-made splints.

This paper focuses on the creation of custom-made splints, since its end use is considered synonymous to fundamental benefits of Additive Manufacturing in terms of Mass Customisation with regards to anatomical fit, function and appearance. Whilst splints can be designed for many purposes, this study focused on wrist immobilisation splints intended to alleviate the symptoms of rheumatoid arthritis as an exemplar application. An image of an immobilisation splint can be found 
in Figure 1. If prescribed by a splinting practitioner, patients are typically provided with wear instructions, including how to don/doff the splint and the expected wear schedule. Each wear schedule is tailored to suit the patient, their lifestyle and the suggested optimal treatment approach to their condition, determined by their therapist (Lohman et al., 2001).

A process model for designing and fabricating a custom-made wrist immobilisation splint can be found in Figure 2, deduced from Lohman (2001), Jacobs and Austin (2003) and Austin (2003b). In summary, custom-made wrist immobilisation splints are typically handmade; they are formed from sheets of thermoplastic which are cut, heated, moulded to the patient, adjusted, then finished with fasteners (such as Velcro) to ensure a secure fit to the patient. Fundamentally, the splinting process is inherently a combination of designing and making in a single process. As a result, limitations of materials and fabrication processes impede the design for fit and function. Consequently, several factors affect patient compliance, such as discomfort and poor aesthetics, often resulting in a reduced willingness to wear splints to match the prescribed wear regime. The aesthetics of splints can have implications on the suggested duration and location of wear (Veehof et al., 2008), since splints typically look clinical and unattractive despite the best efforts of clinicians to finish splints to a high standard and to suit patient preference. For example, patients are encouraged to choose different Velcro colours in a bid to improve compliance (Austin, 2003a). However, choice is limited to the material stock available to the clinic and the associated properties of the thermoplastic.

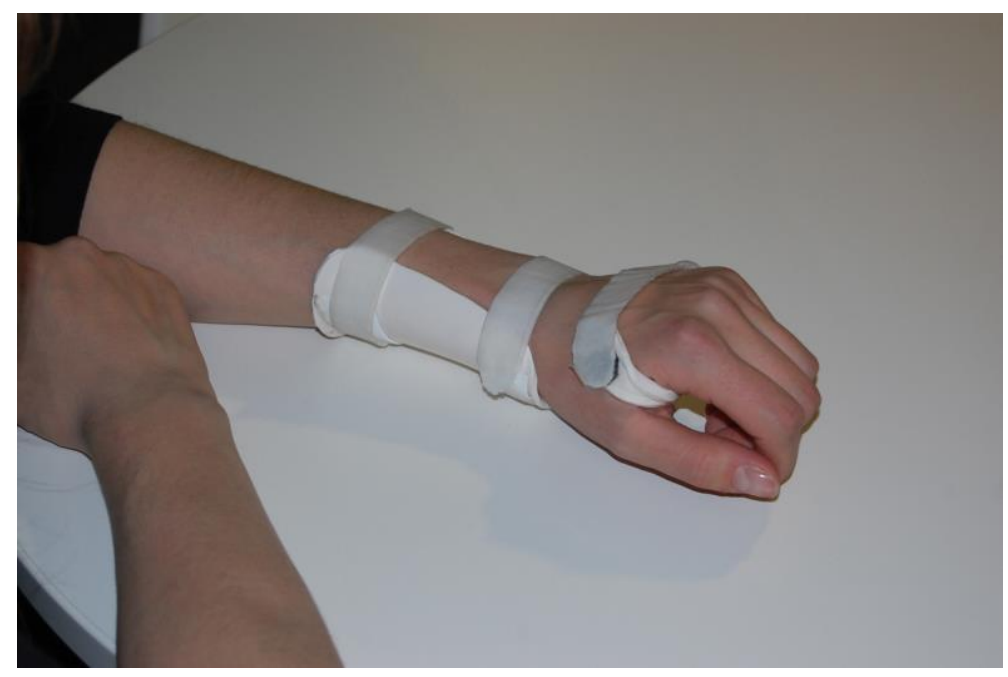

Figure 1. Custom-made wrist immobilisation splint 


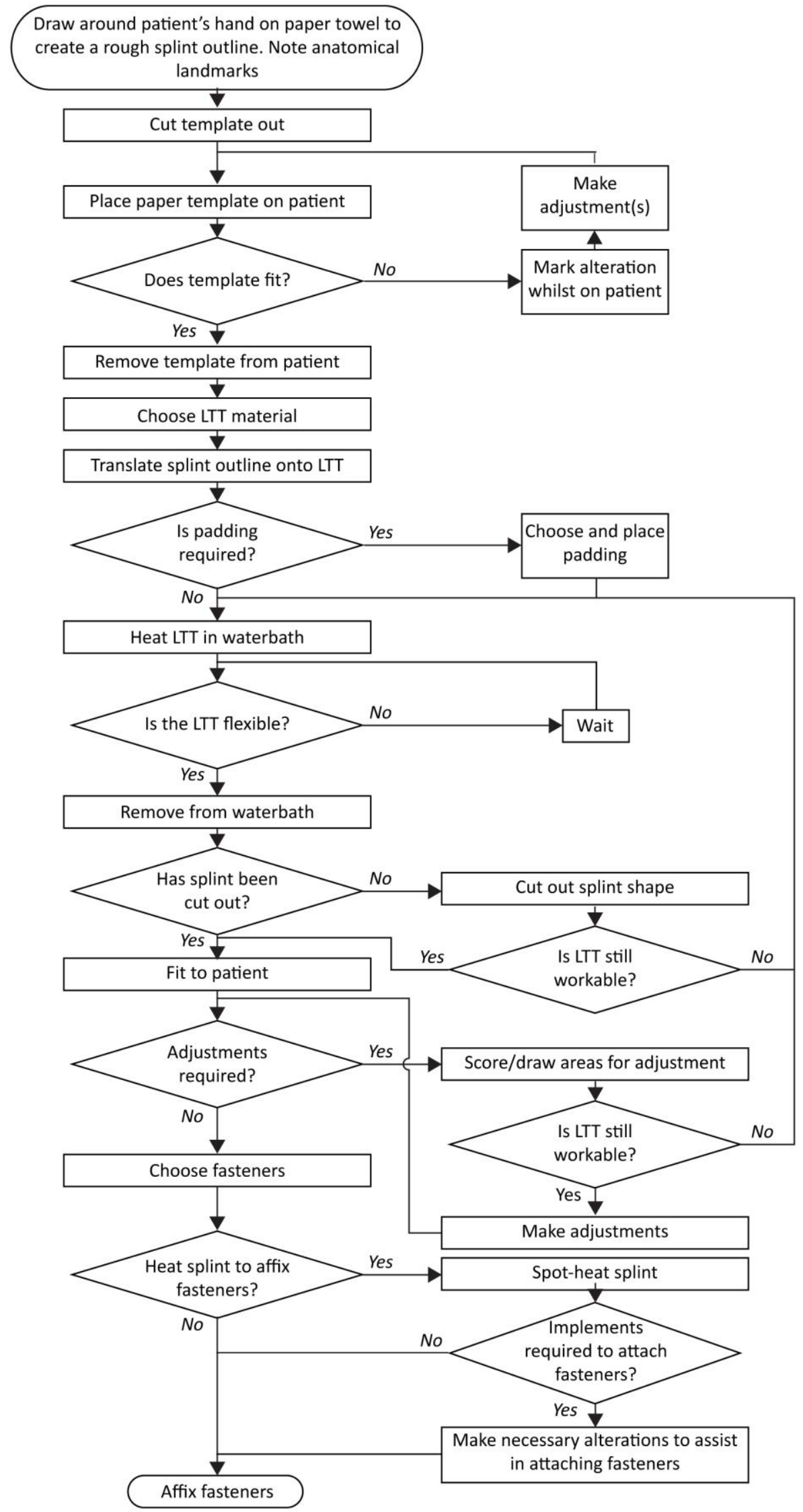

Figure 2. Traditional splint fabrication process (Paterson et al., 2012). Image courtesy of SFF Symposium 
As the manufacturing process is entirely manual and skill dependent, the splint may also be poorly fitted, resulting in shear stress, directional misalignment and pressure over bony prominences, which in turn can induce pressure sores (Coppard, 2001). Furthermore, the presence of a thermoplastic splint with uniform thickness and limited perforation can induce excessive perspiration, which can collect within the porous elements of padding (if present). In turn, this harbours bacteria, resulting in an odorous, unhygienic yet often compulsory form of treatment for patients (Coppard and Lynn, 2001). Furthermore, splints are difficult to keep clean, particularly if a padded lining is present.

In response to these issues, the opportunity for using Additive Manufacturing (AM) for upper extremity splinting was considered a viable option for future splint fabrication. Campbell (2006) states that AM can account for functional, environmental, ergonomic, aesthetic, emotional and user fit requirements, and as such, is a proven viable method for the design and fabrication for customised body-fitting items. The scope for AM applications continues to widen in a broad range of disciplines; the use in the medical and dental industry, for example, continues to be the world's third largest serving industry (15.1\%) in the AM sector for the past 11 years (Wohlers, 2012). AM has already been explored in a range of exoskeletal assistive devices, ranging from clubfoot treatment methods (Gervasi et al., 2009; Smith, 2011b; Cook et al., 2010), spinal braces (Summit and Trauner, 2010a) and ankle-foot orthoses (Gibson et al., 2013; Faustini et al., 2008; Mavroidis et al., 2011; Pallari et al., 2010), the latter being the focus of a Framework 7 European project called A-Footprint. The majority of these works have focused on the use of Laser Sintering (LS); the benefits of which include relatively low part cost compared to other AM processes and the ability to retrieve unsintered powder for future use. Furthermore, the fact that unsintered powder subsequently behaves as support for down-facing structures is also a significant advantage, since this enables the creation of complex geometries without incurring significant clean-up time and subsequent costs associated with manual post-processing, whilst reducing the build time. Additive Manufactured Textiles (AMT) described by Bingham et al. (2007) demonstrate benefits of AM in terms of part consolidation and assembly builds whilst proposing functional articulating wearable structures. Various AMT linkage designs and arrays have been explored for stab-resistant body armour, demonstrating its capabilities in generating functional constructs to enable movement of the intended wearer (Johnson et al., 2013). Furthermore, AMT's look visually appealing, and although their integration into artefacts to date remains a niche topic, the scope for integrated aesthetical yet discrete functional AM textiles into custom-fitting wearable devices is entirely feasible.

Extending the use of AM in context of assistive devices, Gibson et al. (2013) investigated the suitability of a low cost extrusion-type 3D printer; their justification being a low cost, in-clinic approach to fabrication through the use of a RapMan 3D print extrusion system (3D Systems, Rock Hill, SC, USA). Furthermore, Mavroidis et al. (2011) used Stereolithography (SLA) (3D Systems, Rock Hill, SC, USA) for ankle foot orthoses, since a range of materials could be used to offer different properties; for example, Arptech's DSM Somos 9120 Epoxy (Arptech Pty Ltd, Victoria, Australia) offered a flexible solution whilst being biocompatible. SLA also demonstrates a high level of surface quality and resolution when compared to original STL data. In the context of using material jetting systems for fabrication of assistive devices, Smith (2011a) describes the developments of the Miraclefeet organisation and North Design Labs with Objet Connex systems into custom made paediatric clubfoot orthoses. The most significant benefit of the approach was through exploiting the multimaterial build capabilities on offer, enabling heterogeneous builds including a range of Shore hardnesses alongside more rigid materials to incorporate functional parts, such as flexible hinges and soft edge features for improved functionality and comfort.

Despite the previous studies into AM for lower limb prosthetics and orthotics, the suitability of AM technologies in the context of upper extremity (i.e. hand, wrist and forearm) splinting is yet to be 
compared and evaluated, with suitable courses of action established for future development. To date, there has only been limited research and development into AM upper extremity splinting. The application has been implied by Summit and Trauner (2010b), Fried et al. (2004) and Fried (2007), and although no commercial approach has been proposed yet, a number of research institutes and individuals have explored the feasibility of AM splinting. For example, Fraunhofer IPA used an EOS P100 LS system with PA2200 powder (EOS GmbH Electro Optical Systems, Krailling, Germany) to offer a single example of an attractive solution to traditional splinting (Grzesiak, 2010). The benefit of LS in this instance was the ability to introduce intricate locking mechanisms which would have required less post-processing to remove unsintered powder acting as support structures. Whilst Fraunhofer IPA have explored the integration of Voronoi patterns to their prototype (Breuninger, 2010), Evill (2013) has proposed the use of LS for splinting wrist fractures, incorporating a honeycomb structure with interlocking fasteners. Palousek et al. (2013) also explored AM for splinting, but the intent was limited to reverse engineering a single existing splint that had been designed for traditional manufacturing techniques, and this attempt failed to recognise or explore the fundamental opportunities of design for AM principles such as improved aesthetics and or lightweight lattice type structures.

In terms of material jetting technologies, Carpal Skin by Oxman (2010) explored the multiple material build capabilities available with Objet Connex systems (Stratasys, Eden Prairie, MN, USA). A range of Shore hardnesses were incorporated alongside stiffer materials in one build, resulting in a heterogeneous splint. The term 'synthetic anisotropy' was established to communicate the effect of directional influencers in the form of a specified pattern distribution to allow or restrict movement, the dispersion of which was dictated by an algorithm sourced from a pain map defined by an individual (PopTech, 2009; Oxman, 2011). Whilst this approach explores the opportunities of design for AM, no clinical validity has been published to date. Taking an alternative stance to performance, functionality and design strategies of integrated features, Paterson et al. (2012) explored the potential integration of multiple materials into wrist splints under the direction of qualified and experienced clinicians who specialise in the design and fabrication of custom-fitted wrist splints within the National Health Service (NHS) in the United Kingdom. The research focused on developing a specialised workflow designed for splinting practitioners to allow them to design splints in a virtual environment to support AM; the manuscript focused specifically on the intent for placing multiple materials to behave as hinges or cushioned features as opposed to traditional fabrication processes where a similar approach would be impossible to replicate.

Having identified the relative strengths of four different AM systems along with existing work into other custom-fitted devices, the authors chose to explore opportunities in upper extremity splinting for improved fit, functionality and aesthetics. Features which were considered as potentially beneficial included a ' $b$ e $s t \quad f i$ tapprfoachrtosptovide a coostomised fit for a patient relative to their anatomical and rehabilitation needs, and AMT elements for hinges using LS for easier donning and doffing. Furthermore, the authors chose to explore the opportunities for integrating varying Shore hardnesses into wrist splints using the Objet Connex system, to include functional features such as integrated elastomer hinges or cushioned features over bony prominences. These new opportunities were impossible to deliver in traditional splinting, but are now entirely feasible as a result of AM. By exploring new, novel integrated applications into upper extremity splinting, future avenues for product development could be pursued. It was anticipated that with these potential benefits, patient compliance would be improved.

\section{Aim and objectives}

The research reported in this paper represents one stage of a long-term research project exploring the whole process of digital splint design and manufacture, from data acquisition through to data 
manipulation in Three-Dimensional (3D) Computer-Aided Design (CAD) to support AM. This paper describes the exploration of AM prototypes through comparison and subsequent suitability of different AM processes based on the digital design workflow developed and described by Paterson (2013). By investigating previous research activity and potential AM process benefits, several design characteristics were planned for upper extremity splint integration for this investigation. Particular focus was placed on improving aesthetics, fit and function of splints, and by exploring existing design features into the context for upper extremity splinting.

The aim of this paper was to demonstrate and evaluate the suitability of a range of AM processes to deliver custom-fitting wrist splints, using the following objectives:

Objective 1. To evaluate the design and fabrication of homogeneous AM splints.

Objective 2. To evaluate the design and fabrication of heterogeneous (multiple material) AM splints with functional features, e.g. an elastomer hinge and soft edges.

Objective 3. To demonstrate varied part consolidation, resulting from AM processes through integrated fastener features.

Objective 4. To evaluate the integration of a textile-hinge to demonstrate assembly build capabilities.

Objective 5. Establish relative strengths and limitations of each AM process relative to recognised best practice in splint design.

Objective 6. Establish areas for future research and development relating to design of wrist splints for AM. 


\section{Method}

One of the fundamental requirements of the AM splinting approach was to deliver a customised-fit to the patient, since this is a standard requirement in traditional custom-made splinting. To deliver this, the patient's skin surface topography would be required to capture the patient's anatomical data. This data could then be used to extract and subsequently generate a 3D virtual form of a splint to match their topography.

Before AM splints could be fabricated for evaluation, 3D CAD splint models had to be generated. The organic topography of a forearm and hand was required to generate an accurate profile for CAD manipulation. Scan data was acquired from a healthy volunteer by 3D laser scanning a plaster cast, as described by Paterson et al. (2010). A plaster cast was used to eradicate concerns with noise that would otherwise be collected through involuntary movement and tremor during scanning. The plaster cast provided a repeatable, static data source in case a repeat scan was required later in the research process (Paterson, 2013). This form of data acquisition was to enable this research only and is not suggested for future clinical practice. The plaster cast was scanned with a ZCorp ZScanner 800 hand-held 3D laser scanner (3D Systems, Rock Hill, SC, USA). The same scan data was used for all subsequent splint designs, and therefore each demonstrated almost identical anatomical topographies (excluding integrated features such as lattices and fasteners) which would therefore fit the participant used to generate the plaster cast. Using this scan data, six different splints were designed in a range of 3D CAD software packages and plugins;

- Autodesk $^{\circledR}$ Maya ${ }^{\circledR} 2011$ (Autodesk, San Rafael, CA, USA)

- Geomagic Studio 2012 (3D Systems, Rock Hill, SC, USA)

- McNeel Rhinoceros ${ }^{\circledR}$ Version 4.0 (Robert McNeel \& Associates, Seattle, WA, USA)

o Grasshopper plugin (Robert McNeel \& Associates, Seattle, WA, USA)

- PTC Pro/Engineer Wildfire 5.0 (PTC, Needham, MA, USA)

- FreeForm Modelling Plus (3D Systems, Rock Hill, SC, USA)

The justification for using a wide range of CAD programs was related to the underlying research; a separate and previous stage in the overall research project was to develop a specialised CAD workflow that effectively replicated the splint design process used by splinting practitioners/clinicians. Therefore, there was a need to establish suitable tools which could ideally replicate and/or improve upon traditional fabrication methods and techniques in a virtual environment within 3D CAD programs. Several different CAD packages were used during this phase of the research since each program had different tools, offering different strengths and limitations which were considered appropriate for splinting applications. Tools and CAD strategies were developed in that phase of the research to create a customised software workflow and these methods were taken forward to the design and fabrication of the customised AM splints described in this paper. The design workflow and exploration of CAD approaches is described fully by Paterson (2013) and is the subject of publications pending. In short, this workflow translates into a threedimensional CAD environment the same design intent as the traditional design approach. Therefore the "design rules" for the design of splints is adhered to in the digital workflow and this has been evaluated and approved by a number of qualified splinting professionals. The design steps include, for example, defining the boundary of the splint following anatomical landmarks, defining the material thickness, rounding edges and alleviating pressure on sensitive areas. Over and above the traditional approach, the choice of lattice pattern is introduced to reduce weight, improve ventilation and enhance aesthetics. This workflow has been developed to facilitate recognised clinical practice within a digital environment and has been critically evaluated by experienced and qualified clinical professionals.

Four different 3D CAD models were created in total, designed for homogeneous AM fabrication using; 
- Laser Sintering (LS): EOS P100 Formiga, made with EOS PA2200 50:50 powder (EOS GmbH Electro Optical Systems, Krailling, Germany). This process was used to explore opportunities to integrate textile elements into a splint for added functionality.

- Fused Deposition Modelling (FDM): Stratasys Dimension SST1200es, made with ABS (Stratasys, Eden Prairie, MN, USA).

- Stereolithography (SLA): 3D Systems 250, made with Accura Xtreme resin (3D Systems, Rock Hill, SC, USA)

- PolyJet matrix material jetting: Objet Connex 500, made with FullCure ${ }^{\circledast} 515$ and FullCure ${ }^{\circ} 535$ to generate RGD5160-DM (Stratasys, Eden Prairie, MN, USA), displaying ABS-like properties.

Finally, a heterogeneous splint was designed to exploit the multiple material capabilities of Objet Connex technologies, by using TangoBlackPlus and VeroWhitePlus to generate DM9840FLX and DM9850FLX material ranges (Stratasys, Eden Prairie, MN, USA). Although outputs were similar to Oxman (2012), the combination with integrated aesthetic lattice structures was also targeted as an output in this context. With the exception of the Objet Connex heterogeneous build, material choice was not considered important within this investigation since the research focused on highlighting potential differences in $A M$ systems and their specific capabilities. However, the authors acknowledge that material choice is crucial in many aspects in assessing part quality and delivering for the intended need. Material choice to the extent of being able to vary properties in the heterogeneous build was, however, important for the multimaterial splint since this was considered the most important characteristic to display in this context.

In the next section, each of these designs will be described in more detail, corresponding to the AM process considered most appropriate for the design. Different designs were fabricated on different AM systems to demonstrate the versatility of AM systems relative to their particular strengths described in Section 1 (Introduction). Furthermore, LS was only used for the fabrication of a textile splint, since previous studies described in Section 1 have already demonstrated homogeneous splint prototypes using LS; the development of a textile element in this case was therefore considered a potential novel contribution to knowledge.

\subsection{Homogeneous AM Textile splint}

The AM Textile splint featured an AMT element along the ulnar aspect. This was incorporated to consolidate splint parts into one assembly. The AMT element was designed to behave as a hinge to enable the user to open the splint for easier donning and doffing. Furthermore, the AMT element was designed to follow contours of the upper extremity geometry, demonstrating the drapability and free movement described by Bingham et al. (2007). The repeating units in the AMT element were formed to follow the topography of the scan data, and were aligned using a custom mesh array algorithm devised by Bingham and Hague (2013) in MATLAB ${ }^{\circledR}$ (MathWorks, Natick, MA, USA). The textile element was then incorporated into the remaining splint geometry using uniform spaced links generated in Grasshopper; a generative modelling plugin for Rhinoceros to enable quick adjustments within set parameters. The automated linkages were united with the main structures of the splint using a Boolean Union function.

The AMT element was specifically designed to exploit the freedom of form available through LS (Figure 3), since support structures commonly required by other AM systems would not be required in this instance. Since un-sintered powder provides support for overhangs, LS was considered the most effective approach since overhangs in AMT linkages were abundant. Clean up time was also considered, since the un-sintered powder would only require removal via high-pressure air jets and vibration to remove excess powder between linkages. Other processes could be used such as 3D jetting / printing techniques for example. However, depending on the exact process and material 
combination used, support material removal could result in additional time and in some cases could damage the AMT linkages. SLA would also prove ineffective for this approach because supports would be required for links within the textile element which would prove difficult and time consuming to remove.

In addition, mushroom-like fasteners were integrated into the design to demonstrate part consolidation of fasteners within the build, as opposed to detached fasteners used on traditional splints such as Velcro.

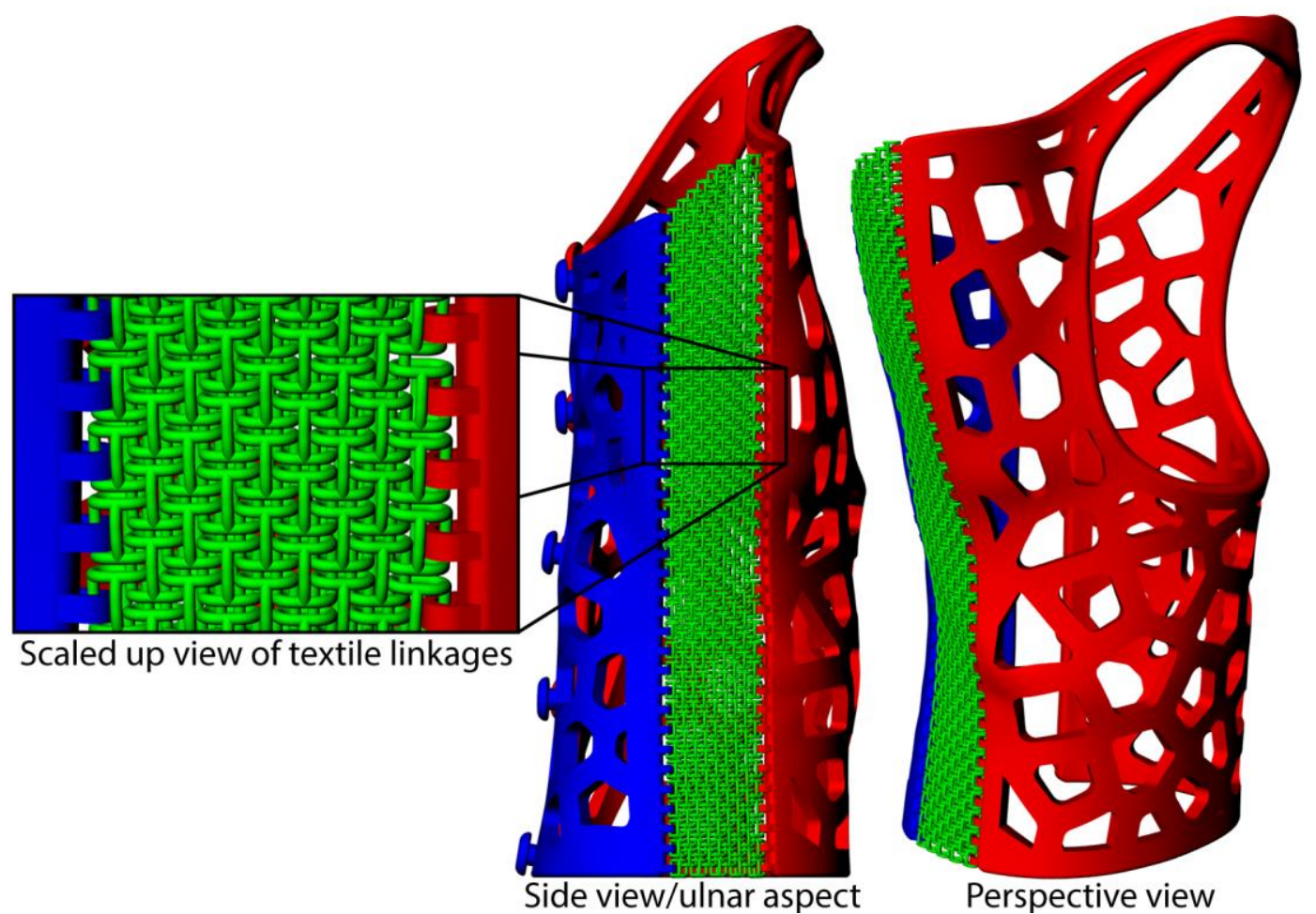

Figure 3: Splint prototype with textile hinge, colour-coded to represent grouped manifold sections. Modelled using Matlab, Rhino and Grasshopper for linkage integration.

\subsection{Homogeneous circumferential build designs}

Two circumferential splint designs were modelled in 3D CAD (Figure 4). The splints were designed in two corresponding parts to enable donning and doffing. The design was intended to behave as a 'pinch-splint', where the user could pinch the palmar region laterally to separate the two halves when taking the splint off. This pinch design demonstrated the ability to integrate subtle, discrete fasteners into the splint whilst still being functional, subsequently highlighting part consolidation compared to traditional fastening methods (e.g. Velcro, D-rings). 

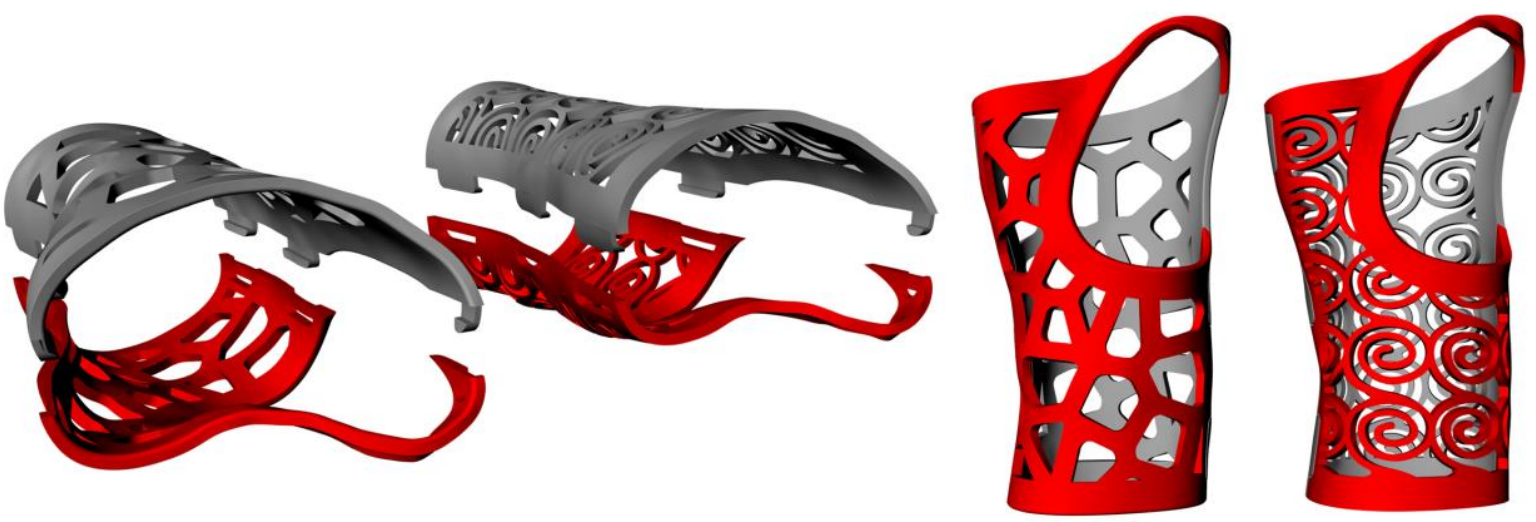

Figure 4: Circumferential, homogeneous two-part splints for SLA (Voronoi) and Objet ( ‘ S ubiilds Iresp£ctively

\subsection{Heterogeneous splint using Objet Connex Technologies}

A splint was designed for heterogeneous AM system fabrication, as described by Paterson et al. (2012). The underlying intent of this approach was to enable the practitioner to specify and localise areas where softer materials might benefit the patient to relieve pressure (e.g. elastomer elements over bony prominences or areas prone to pressure sores) (Paterson, 2013). In traditional splinting, practitioners may have to create cavities over bony prominences or integrate separate gel discs (Coppart and Lynn, 2001), but this approach affects the topography and subsequent aesthetics of the splint. However, the use of the Objet Connex would enable subtle integration of elastomer features which would not drastically affect the topography of the splint, therefore creating a less cumbersome appearance which may be more conducive to complying with wearing regimes for the patient.

Figure 5 shows the 3D CAD model developed specifically for the Objet Connex 500 system, to exploit its multimaterial capabilities. Various closed shells were required within the 3D CAD model to allocate different digital materials prior to fabrication. The shells were created by trimming the initial scan mesh (generated from cloud data) in Geomagic Studio before manipulating and thickening the geometry in other CAD software such as McNeel Rhinoceros. Elements labelled ' 1 ' were soft elastomer edges to provide a comfortable interface between the skin and the rigid splint structures (labelled ' 2 '). A soft elastomer cushion (3) was located over a bony prominence (pisiform), and a flexible hinge (element 4 ) was integrated along the ulnar aspect of the splint to enable donning and doffing. 


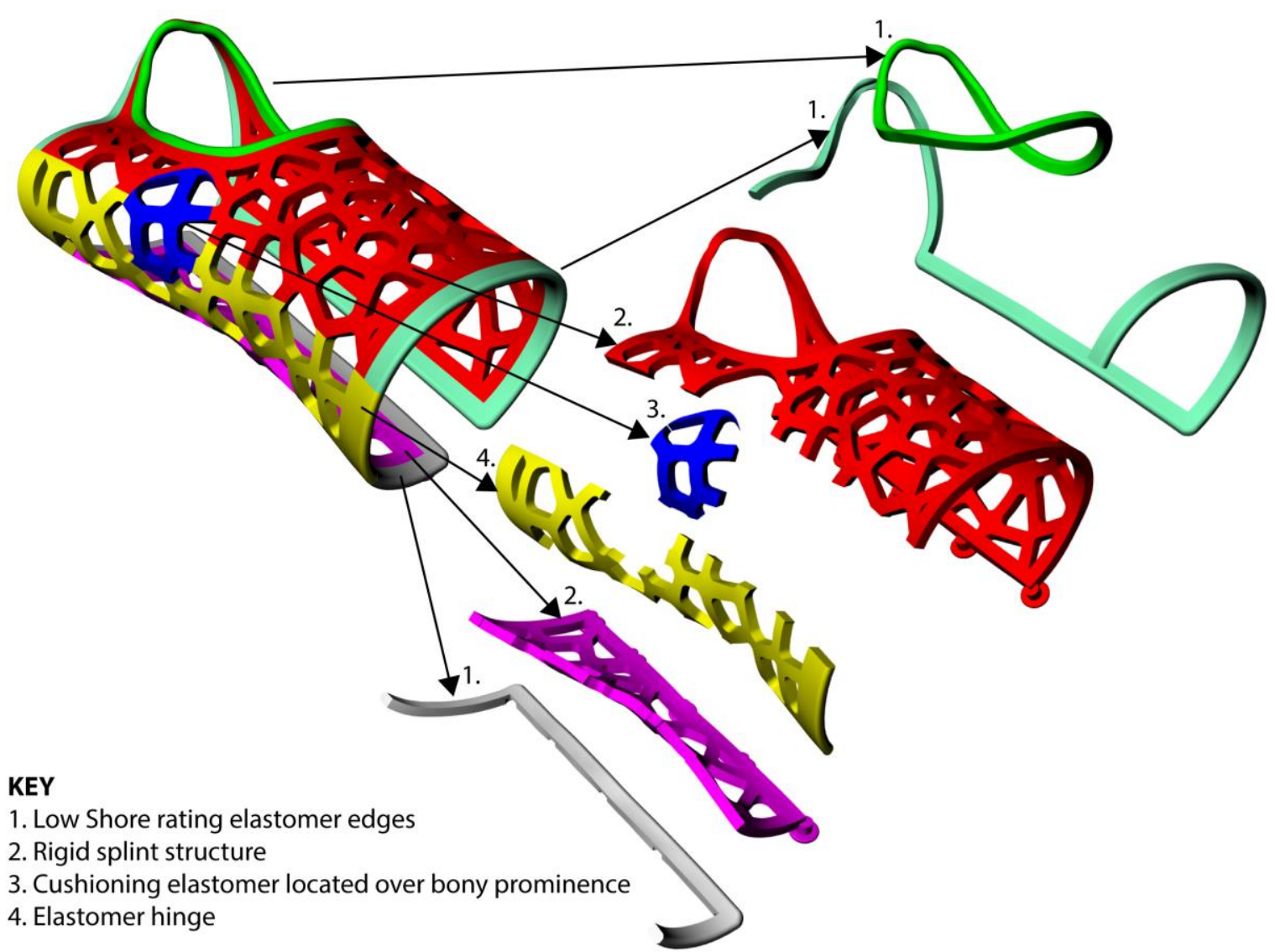

Figure 5: Closed shell distribution and intent of heterogeneous wrist splint

\section{Results}

All of the splint prototypes were built according to the suppliers' recommended parameters using commercially available materials and machines. Each AM process and subsequent build have been reviewed in the following sections.

\subsection{Homogeneous AM Textile Splint}

The LS splint shown in Figure 6 proved successful in capturing its intended outcome of an integrated AMT hinge to enable easier donning and doffing; the links offered sufficient freedom to enable this. The links proved strong enough during a preliminary wearer trial to maintain their structure without failing when the splint was worn. Furthermore, the AMT element added a unique aesthetic quality to the splint. The union of $A M$ textiles and upper extremity splinting with the aim as a medical intervention was considered a world-first.

However, a small number of links remained fused together due to residual unsintered powder trapped between linkages. It is anticipated that the porous nature of the surfaces would also inherently affect the hygiene of the splint by absorbing dirt, sweat, sebum, dead skin cells, etc., as described by Bibb (2006). Furthermore, the AMT element exacerbates these concerns. The small links and tight textile design used in this example could also potentially catch on vellus and/or terminal arm hair, causing discomfort if extracted from hair follicles (i.e. trapping and pulling out body hairs). However, larger links would reduce this risk. Cleaning such a splint would also prove problematic unless immersed in a detergent or washed with an automated process/system such as a dishwasher, as proposed by Fried (2007). LS parts can withstand dishwashing and this has been discussed with reputable LS suppliers, although to date this has not been rigorously tested or 
reported. As a preliminary test, the researchers placed the splint in a dishwasher at various temperatures $\left(45^{\circ} \mathrm{C}, 50^{\circ} \mathrm{C}\right.$ and $\left.65^{\circ} \mathrm{C}\right)$ along with branded dishwasher detergent, with no visible aftereffects.
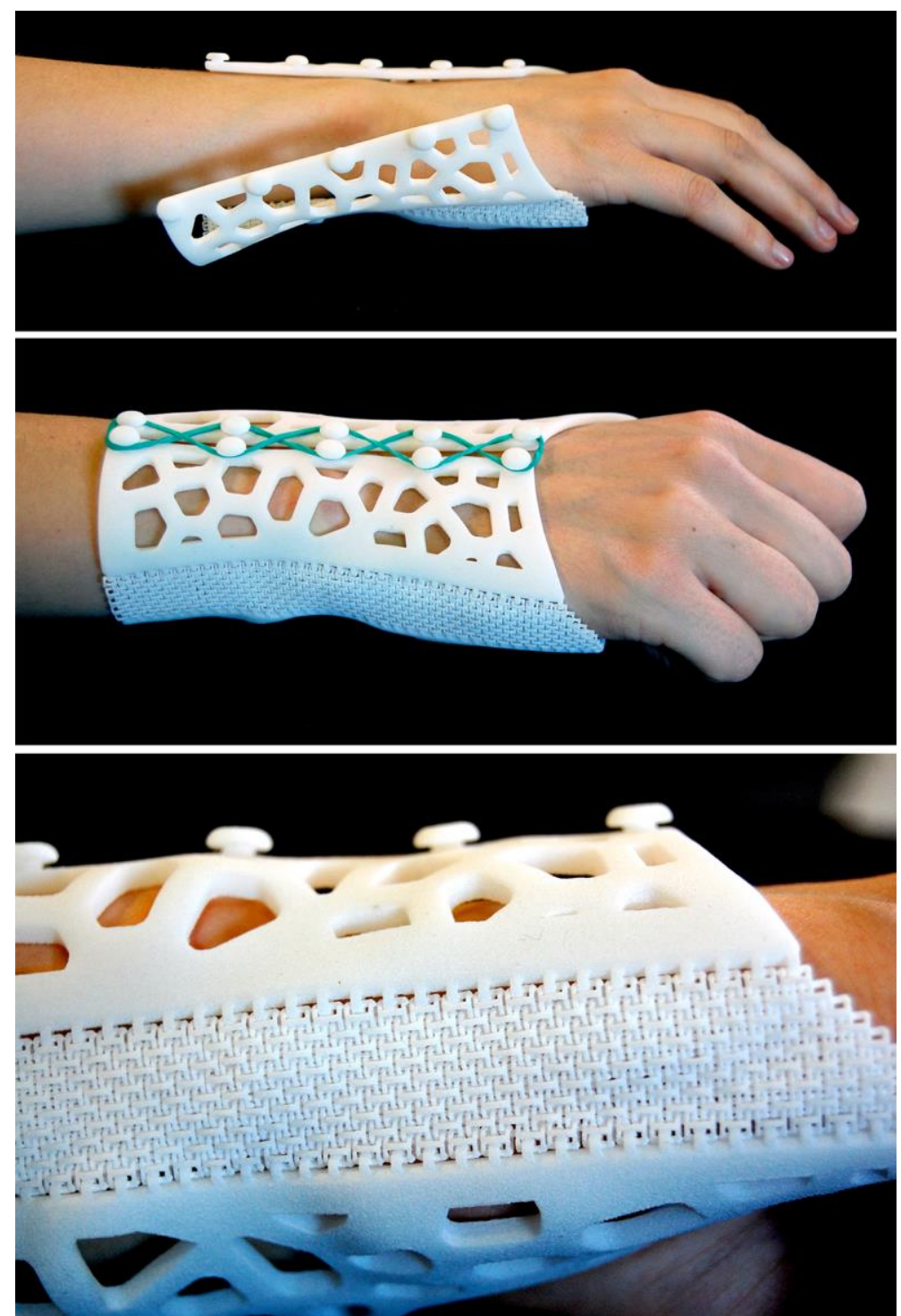

Figure 6: Laser sintered splint with AMT linkage hinge. Prototype courtesy of Dr. C. Majewski and the University of Sheffield

\subsection{Homogeneous circumferential splints}

The FDM splint shown in Figure 7 demonstrated comparatively poor surface quality, with obvious layering and stepping; these factors affected the aesthetics, which also could affect the comfort of the splint at the edges. Pitted areas between layers and tracks could collect waste products as described earlier, and therefore could be an unhygienic solution for a splinting application. However, the ABS material is relatively robust and a widely used material in domestic and wearable products such as frames of eye glasses, and subsequently it is reasonable to anticipate that the ABS splint can withstand mechanical cleaning with mild detergents. 


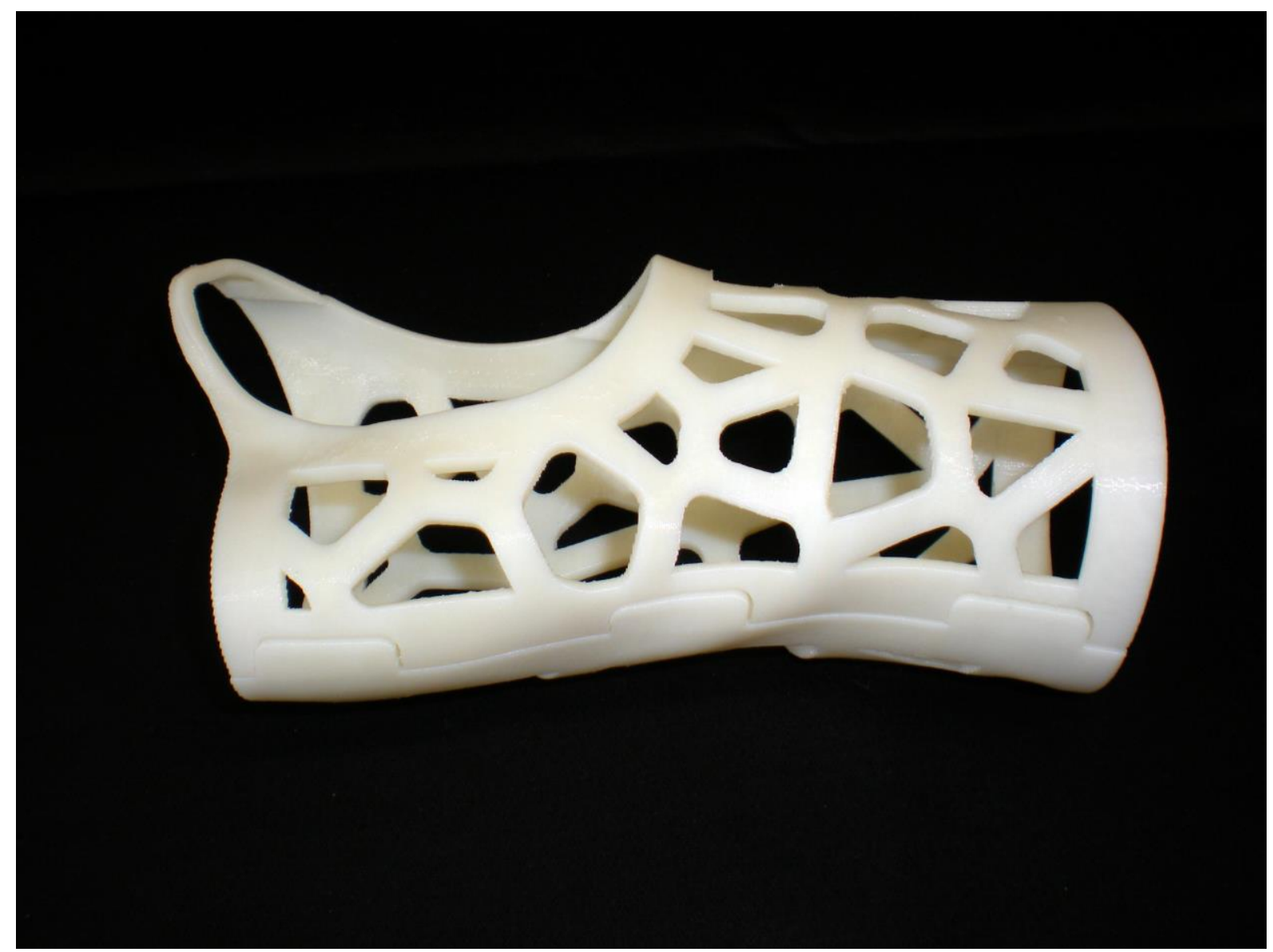

Figure 7: FDM splint

The SLA splint shown in Figure 8 was oriented to reduce the requirements for supports as can be seen in Figure 9. Similar to the homogeneous ABS-like splint, the design of the splint was effective in allowing donning and doffing by pinching the palmar element. Overall, the surface quality of the SLA splint was considered the highest of all the AM processes used in this investigation. The smooth surfaces facilitate cleaning and minimise hygiene risks. However, despite side and up-facing surfaces being smooth, down-facing surfaces demonstrated abrasive imperfections where supports had been removed (Figure 9). Such imperfections could cause discomfort for a patient if left untreated. Manual post-processing with abrasives would be required, as described by Bibb et al. (2009), adding cost in labour and resources if the approach were implemented for clinical application. However, this could be minimised by using patterns that formed self-supporting structures which would eliminate the majority of the supports and the issues encountered in their removal. A simple example is shown in Figure 10. The disadvantage of this approach would be a reduction in aesthetic possibilities and limited patient choice of pattern. 


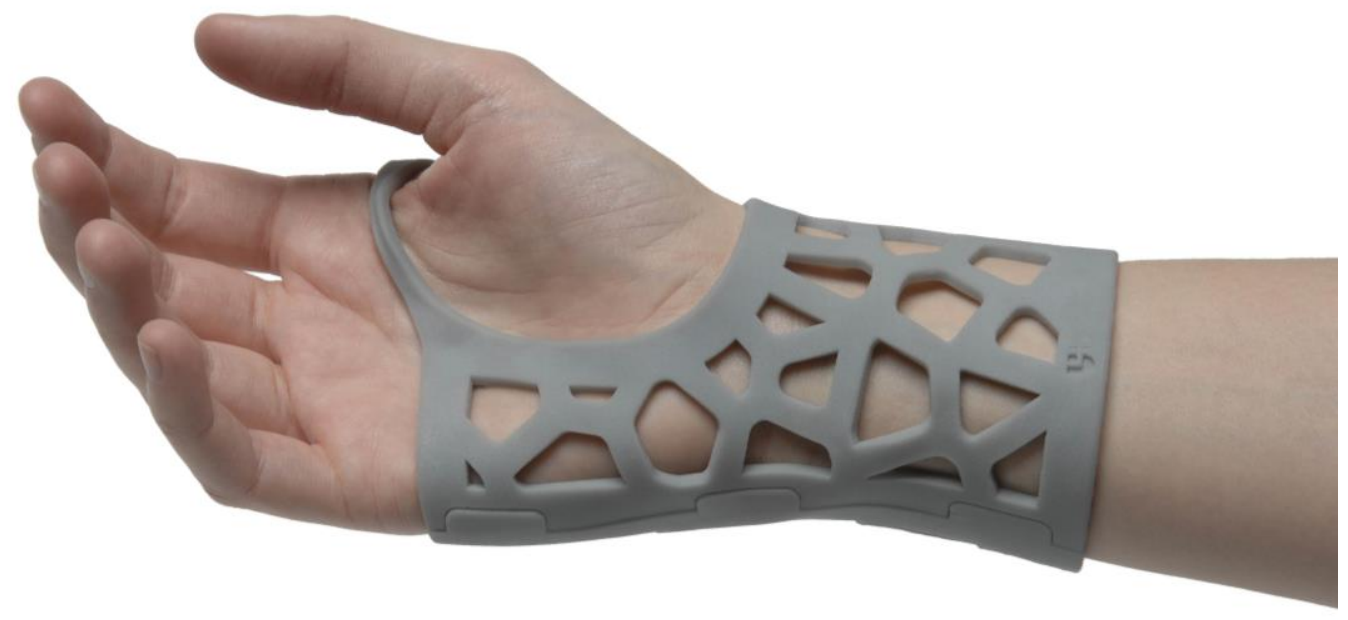

Figure 8: Accura ${ }^{\circledR}$ Xtreme splint, built on a 3D Systems 250 (Paterson et al., 2012). Prototype courtesy of Dr. D. Eggbeer, Product Design Development Research (PDR), Cardiff. Image courtesy of SFF symposium. Image courtesy of SFF Symposium 


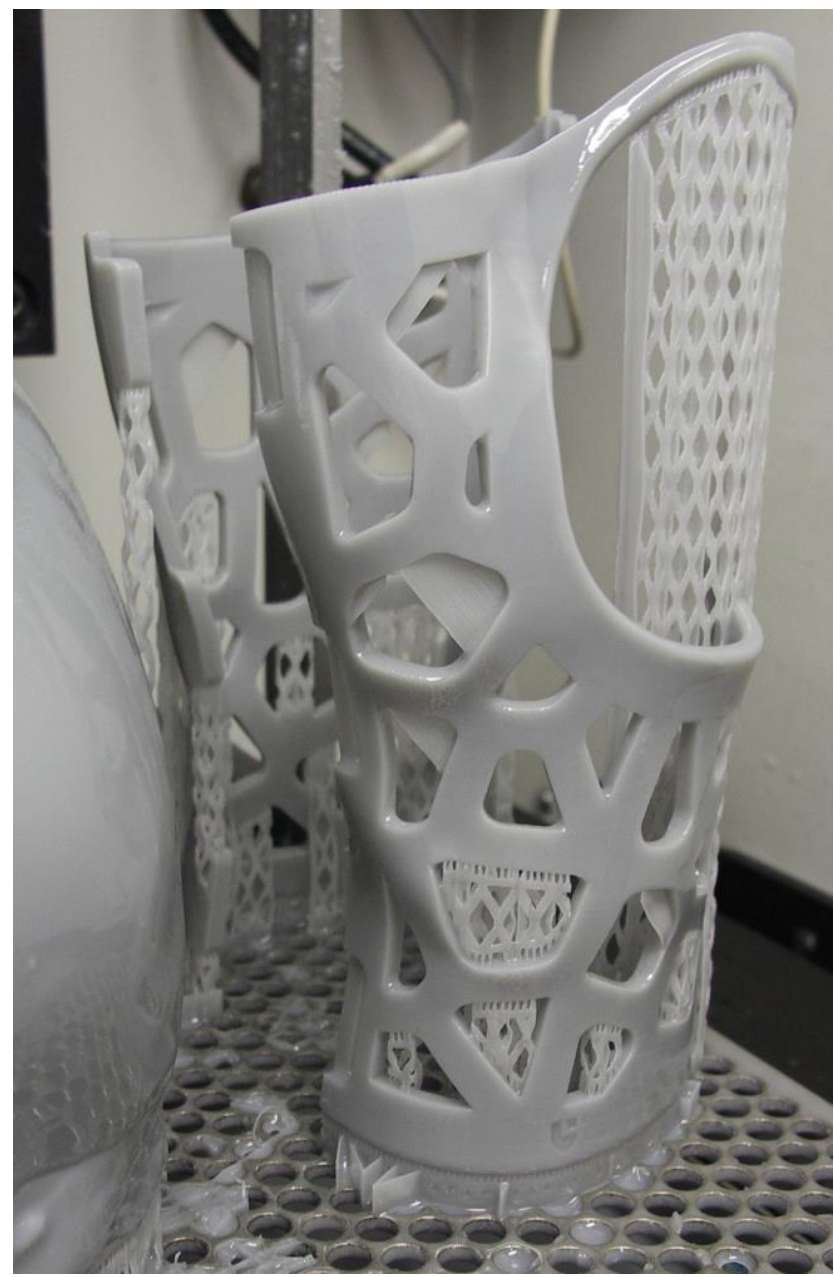

Figure 9: SLA build, showing support lattice structures. Image courtesy of S. Peel at PDR, Cardiff

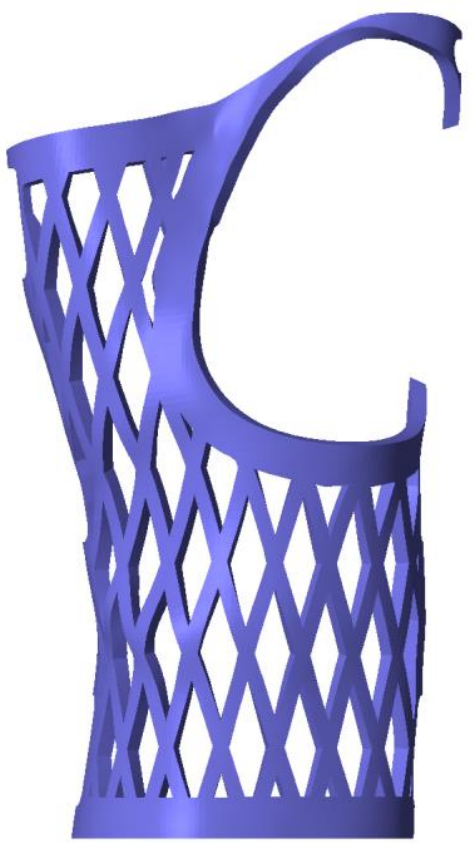

Figure 10: Self-supporting pattern design for SLA 
Finally, a fourth homogeneous splint built on the Objet Connex is shown in Figure 11. An initial visual and tactile interpretation of surface quality was considered acceptable compared to other AM processes described in this paper. In addition, the fastener design and overall splint structure was able to perform and withstand its intended function in flexing to allow the user to don and doff by pinching the palmar aspect of the splint, and subsequently demonstrated part consolidation in the context of AM splint fabrication. However, a significant amount of support material (FullCure 705) was required, but was removed easily and relatively quickly with a high pressure water jet.
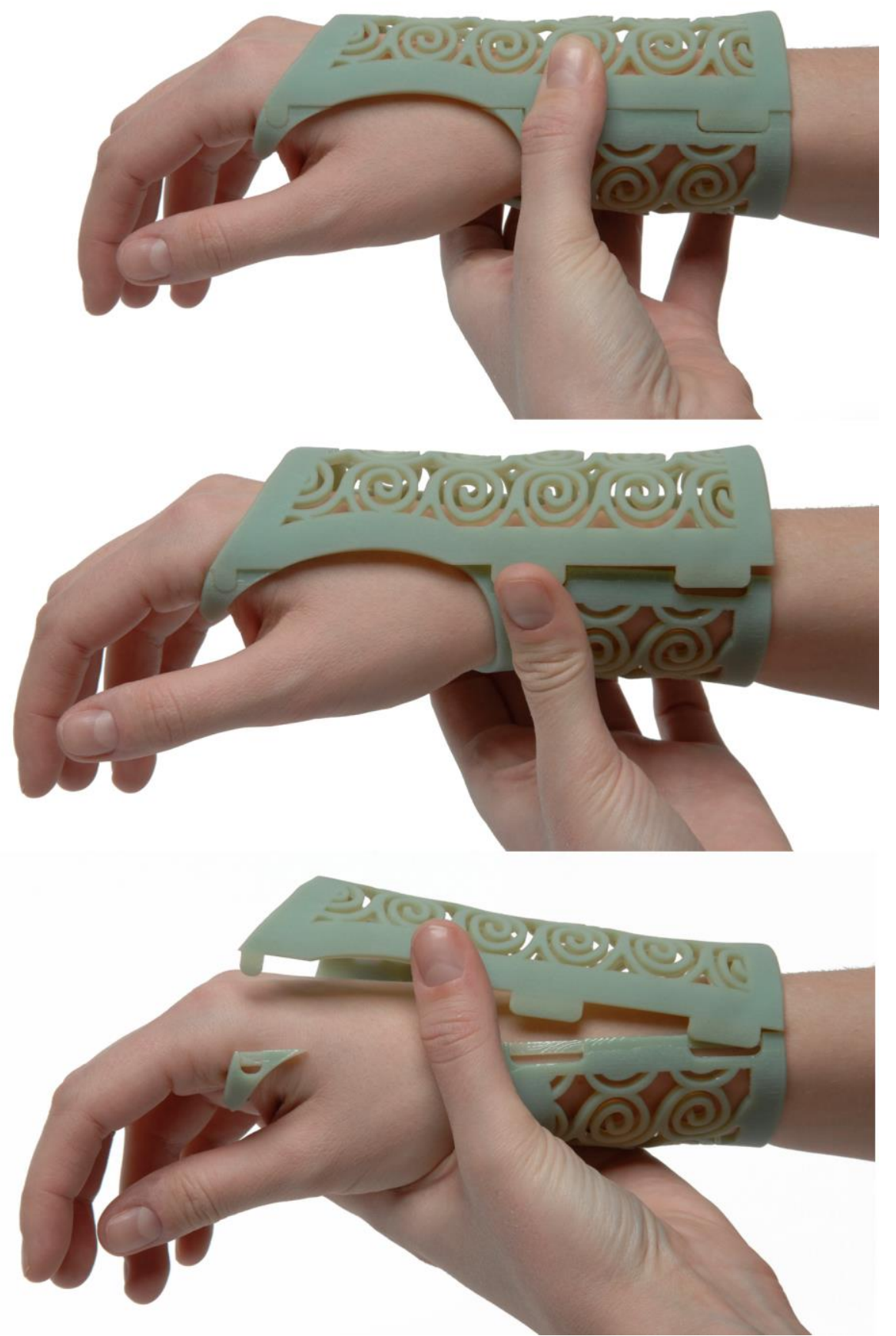

Figure 11: FullCure ${ }^{\circledR 515}$ /FullCure ${ }^{\circledR 535}$ with ABS-like properties, built on the Objet Connex 500 model 


\subsection{Heterogeneous splint using Objet Connex Technologies}

Prior to the building of this example, material choices were specified in Objet Studio ${ }^{\mathrm{TM}}$ (Stratasys, Eden Prairie, MN, USA). The software interface was used to define variables for Objet Connex build systems. The splint made with the DM98 material range provided a stronger colour contrast, as shown in Figure 12.

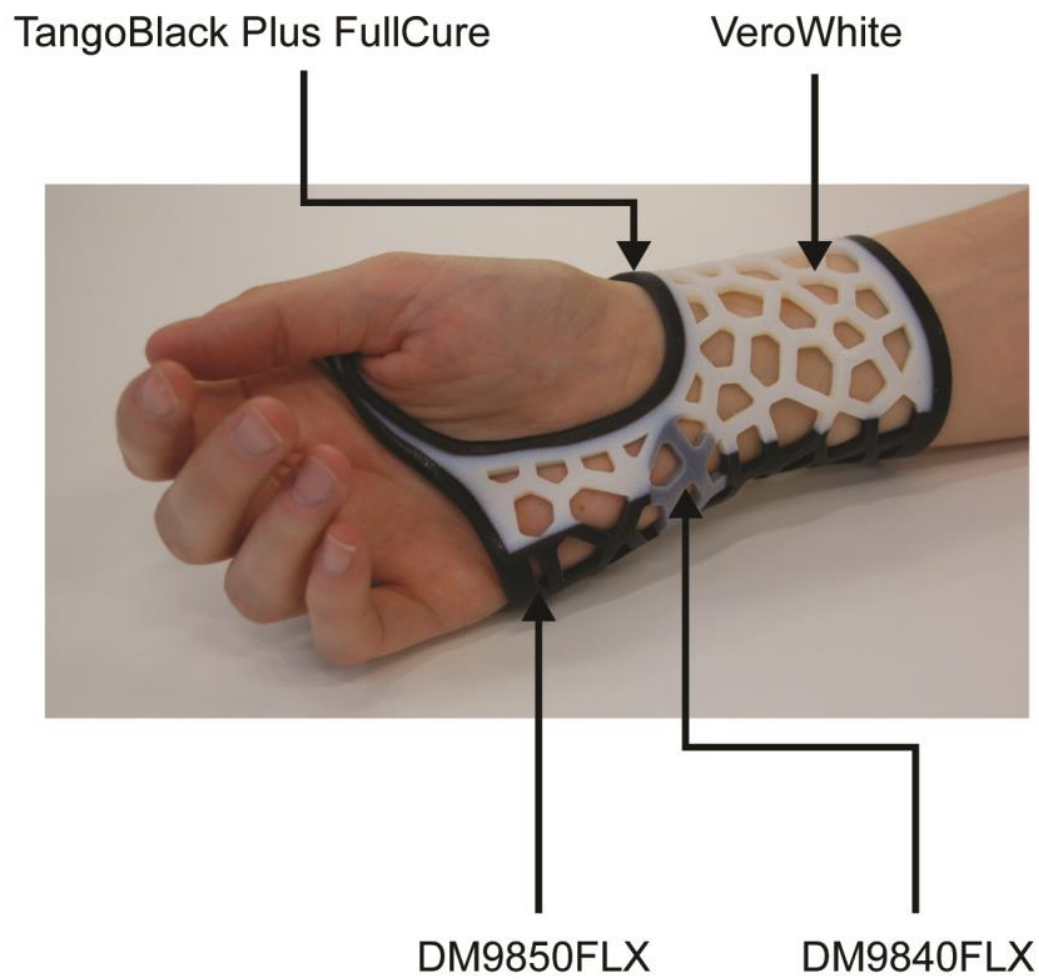

Figure 12: Heterogeneous splint; assorted materials within one AM build, using the Objet Connex

The 9850 Shore 50 elastomer placed over the bony prominence (pisiform) expanded when pressure was applied within the splint, which demonstrated that a small amount of expansion could accommodate swelling if required. The hinge was also functional, although several failures occurred over a period of twelve months (Figure 13). This was due to repeated flexion when opening and closing the splint. The splints were used as proof-of-concept prototypes, and therefore have been handled by a large number of people to demonstrate the intent of the research. This particular splint prototype has been handled by a large number of individuals in presentations and demonstrations and consequently has undergone more than 50 openings and closings over a 12 month period. Many people handled the prototype with little knowledge of its physical limitations and it is possible that its physical limits were exceeded by careless handling. In addition the prototype has been displayed in exhibitions and has been subject to extended periods of exposure to strong light sources which may have affected its physical properties over time. It was also noted that the position and shape of the flexible hinge was not ideal. The position was along the ulnar aspect of the forearm, and the intended wearer had to use significant force whilst adjusting the posture of their hand and wrist to don the splint correctly. Simultaneously, a significant amount of force was applied to the hinge whilst in an open position whilst donning, and therefore the elastomer elements were more susceptible to compression and tension forces resulting in split lines. An additional hinge could have been placed along the radial aspect so both sides of the splint could have been opened to help donning. Similarly, the shape of the splint hinge was inappropriate as it was formed in 3D CAD by two parallel planes intersecting the splint geometry. If one considers a living hinge in a polypropylene DVD case, for example; rotation around a single axis results in a uniform distribution of compression and tension exerted throughout the length of the hinge structure. However, because 
of the organic topography of the splint, compressive and tensile stresses varied throughout the structure, subsequently having a higher tensile concentration towards the borders of the splint, whilst a higher compression concentration was demonstrated on the inner region of the hinge. Therefore, the shape of the hinge element may have benefitted from being a varied shape to suit the topography. Although not formally documented, a very slight level of creep was also observed over time, resulting in a slight twist in the splint. This is most likely due to the fact that the splint has been stood upright on display for extended periods and it would be reasonable to assume that were the splint worn for extended periods it would be more likely to retain the intended shape. Therefore, future research would be required to assess the extent of creep, as well as establishing design rules to reduce local strain and therefore avoid failures at the hinge. However, surface quality and resolution was considered adequate in comparison to FDM and LS.

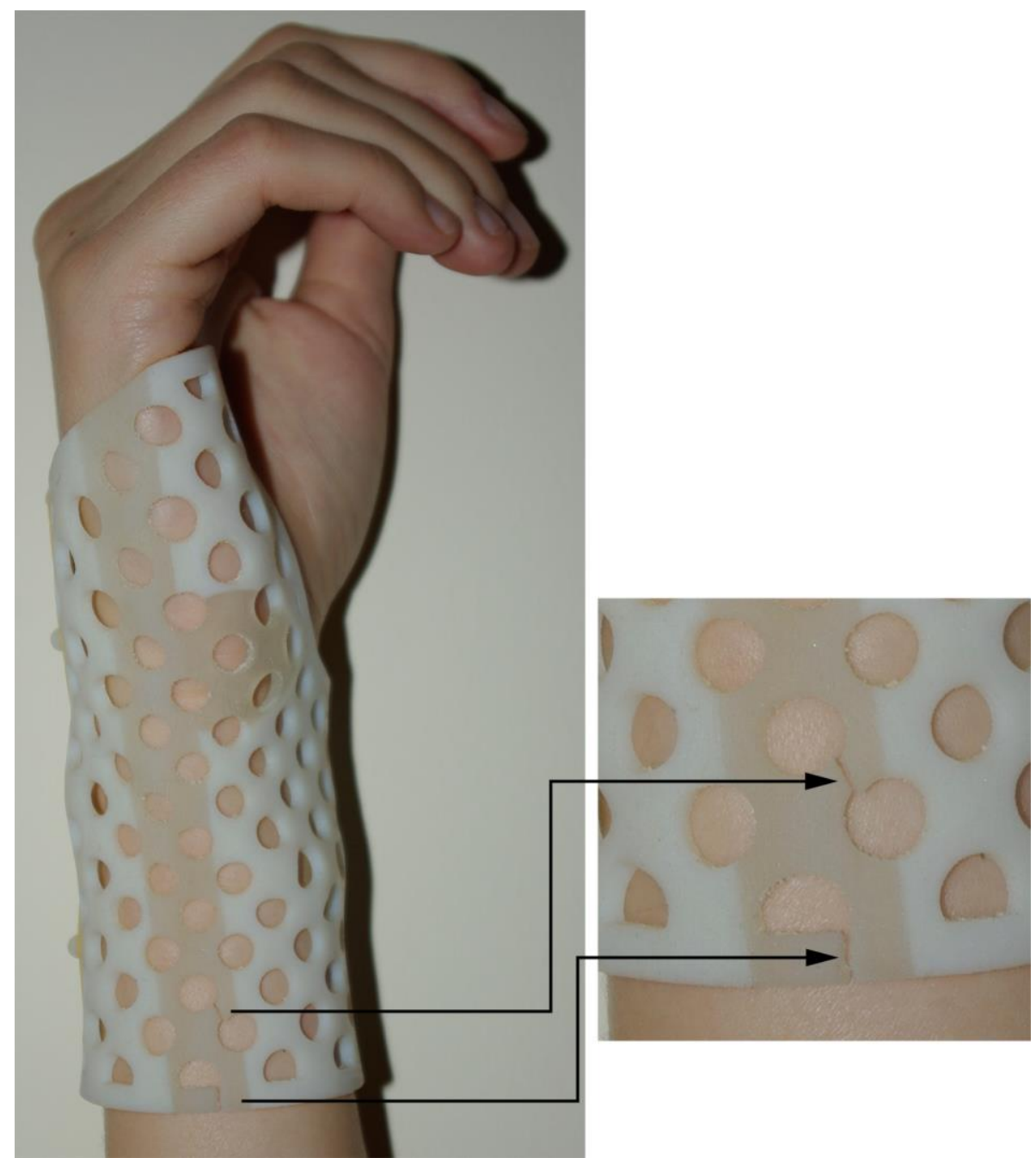

Figure 13: Failures in the multimaterial elastomer hinge (Paterson et al., 2012). Image adapted, courtesy of SFF Symposium

Unfortunately, the multimaterial build required a large volume of support material (FullCure 705), as shown in Figure 14. Much like the ABS-like splint, the support material was removed with a highpressure water jet. Not only does this increase cost in terms of material consumption, but also costs relating to labour time. 


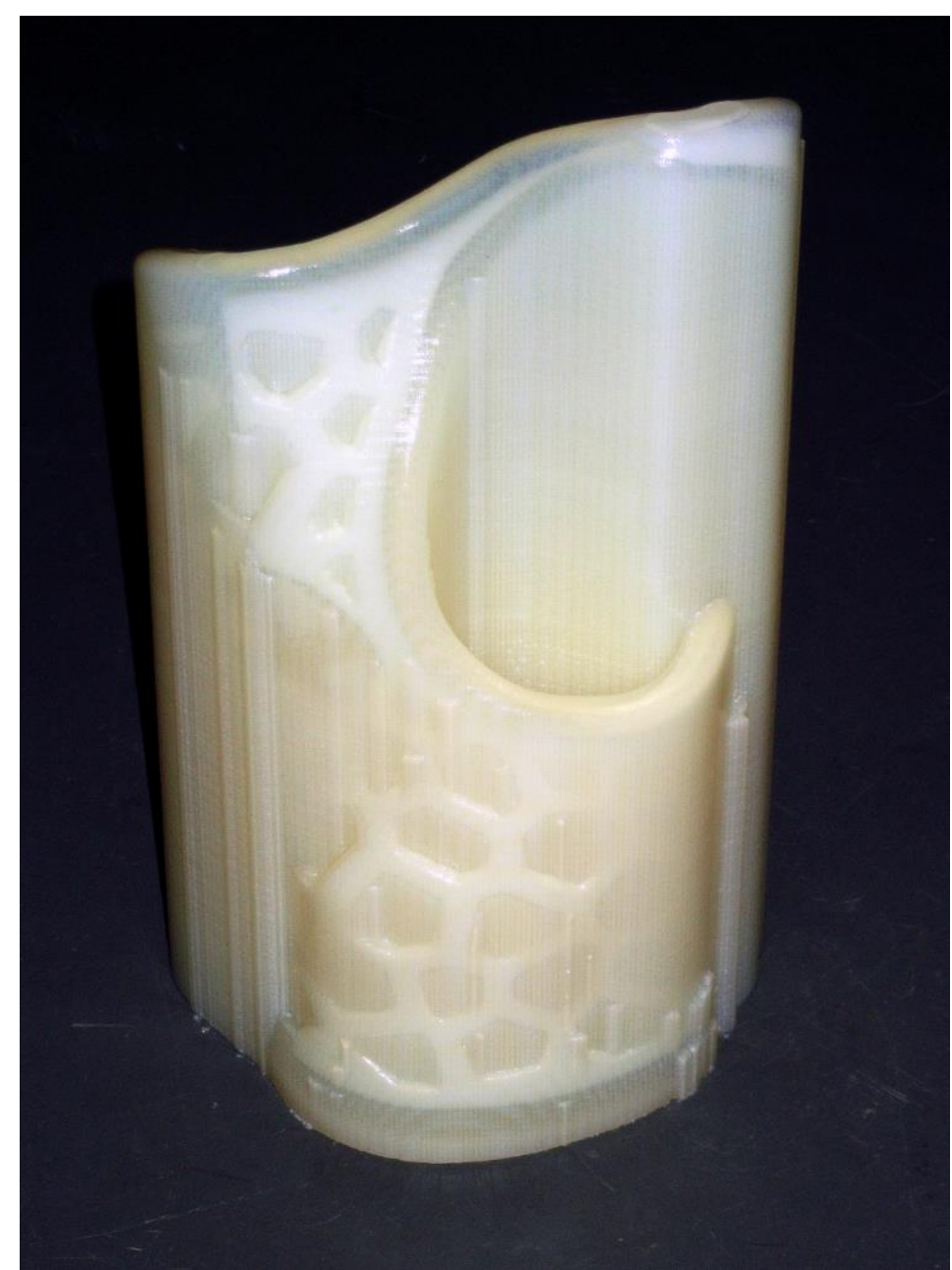

Figure 14: Support material required for the Objet multi-material build in upright position

Table 1 compares evaluation criteria relative to each AM process used within this investigation.

Table 1: Summary of AM processes and comparative evaluation criteria

\section{Conclusions and future work}

Each process displayed benefits and limitations in the context of upper extremity splinting. When compared, the most inappropriate AM process was considered to be FDM. Despite the advantages of robust materials, improvements in surface quality would be needed. SLA proved to have good surface quality and reasonably robust materials, the effects of cleaning notwithstanding. LS and Polyjet material jetting each displayed unique advantageous characteristics, made feasible by the AM process. Previous studies and prototypes which used LS such as Fraunhofer IPA (Grzesiak, 2010) and Evill (2013) demonstrated the ability to integrate aesthetically pleasing structures, but incorporating a textile element has not previously been reported in the context of splinting. This paper further describes additional features which could be beneficial in the future. In addition, the multimaterial splints do demonstrate similarities to Oxman (2010). However, the underlying ethos of placing different materials was a different approach, allowing clinicians to specify materials with varying Shore hardness where they would consider clinically appropriate, whereas Oxman (2010) developed an automated approach to integrate materials to direct or restrict the patient's 
movement. Both strategies demonstrate strengths, and ideally would both be made available features in specialised 3D CAD for clinicians to explore in the future.

In terms of integrated functionality, the heterogeneous splint was the most versatile, and could be exploited in a range of situations, as highlighted by Paterson et al. (2012). If the digitised splinting approach were to be introduced as a realistic option for clinics, the choice of AM process would be dependent on the needs of the patient, as prescribed by their therapist, but a thorough understanding of different $\mathrm{AM}$ processes and relevant AM materials would be needed by the therapists.

Despite the aesthetic and functional advantages displayed in the results, several developments would be required before such processes may be feasible for adoption in clinical situations. Firstly, the development of suitable materials would need to be explored further, taking into consideration the long-term exposure to the skin. Although a number of AM materials such as Objet's MED610 ${ }^{\mathrm{TM}}$ transparent, rigid material and Stratasys' ABS-M30i claim ISO 10993 (Biological evaluation of medical devices) and/or USP 23 Class VI approval in a range of conditions such as irritation and hypersensitivity, regulations involve standardised tests which may not necessarily take into account specific design and manufacturing processes. Whilst this certification demonstrates that the materials are inherently low in toxicity the requirements of the European Medical Device Directive and the various international equivalents require clinical trials that prove the safety of the entire design, materials and manufacturing process.

Cost analysis must also be performed to determine which process, if any, can be cost effective in terms of clinical demands. Although current practice involves the use of cheap materials the labour costs could be cut dramatically. This is especially the case when fabricating duplicate splints, for example. In current practice the entire crafting process must be repeated for each and every splint there are no economies of scale. When using AM, repeat splints would incur materials costs only. It is also important to consider the hidden costs of time and travel involved in clinic appointments for the patient as well as the clinician. The AM process can eliminate repeat prescription time; if a patient requires a replacement/duplicate splint as a result of previous failure or the desire for a different aesthetic design, the patient would not need a repeat clinic session with their practitioner in order to fabricate a new splint. Instead, a request could be logged, and a duplicate splint ordered instantaneously. Such an order could be added to a queue for manufacture, and then dispatched when the build is complete; a similar approach to the latter stages of the proposed automated process by Fried (2007). This reduction in clinic time could reduce demands on the clinic, potentially reducing waiting times and patient waiting lists. In turn, this could improve patient satisfaction in the healthcare system. The economic advantages of an AM approach are predicated on replacing a high labour cost manual crafting process with a much more efficient design then manufacture process. Consequently the economic factors are very much context dependent. It can be envisioned that in high labour cost regions the AM approach may greatly reduce labour costs to the extent that the higher material costs of AM are more than compensated for. This work has been conducted within a UK National Health Service context but future work on costing will enable a more direct calculation of economic benefit in different contexts around the world. Similar arguments for the potential benefits of a digital design and manufacture process have also been explored in maxillofacial prosthetics by Eggbeer (2012). Another implication in cost effectiveness is improved compliance, leading to improved patient outcomes in the longer term. The impact of patient involvement (i.e. choosing patterns and colours) and enhanced aesthetics on compliance is the subject of current research by the authors.

One of the aesthetic considerations available in clinics, which could not be demonstrated fully, was the customisation of colour choice. Colour ranges are currently limited in AM processes; the 3D 
Systems ZPrinter 450 (3D Systems, Rock Hill, SC, USA) can provide multicolour builds, whilst a range of FDM machines can offer various single colour builds. However, within the scope of this investigation, colour choice amongst LS, SLA, FDM and PolyJet material jetting is limited. The Objet Connex gave the widest variety, allowing for an integrated range from black to white. Other colours are achievable with the Objet Connex such as VeroBlue (RGD840), green (ABS-like RGD810) and transparent (VeroClear RGD810) (Stratasys, Eden Prairie, MN, USA), although Holmes (2012) speculates multicolour build capabilities in the future in response to work by Oxman (2012). Some service providers are able to supply colour dyed LS parts but typically colour choice is limited and colour fastness is an issue.

It was hypothesised that the mechanical failures of the multimaterial splints were due to a number of factors, including:

- Limited tensile strength, elongation and tear resistance of flexible Objet materials, e.g. 0.51.5MPa, $150-170 \%$ and $4-6 \mathrm{Kg} / \mathrm{cm}$ respectively for FLX9840-DM material (figures according to Stratasys, 2013). Similar issues were highlighted by Moore and Williams (2012) and Eggbeer et al. (2012) (Figure 15).

- Degradation of material over a period of time (including creep).

- Suboptimal location/shape of the flexible hinge relative to the organic geometry of the splint, and the force exerted when opening and closing the splint when donning and doffing.

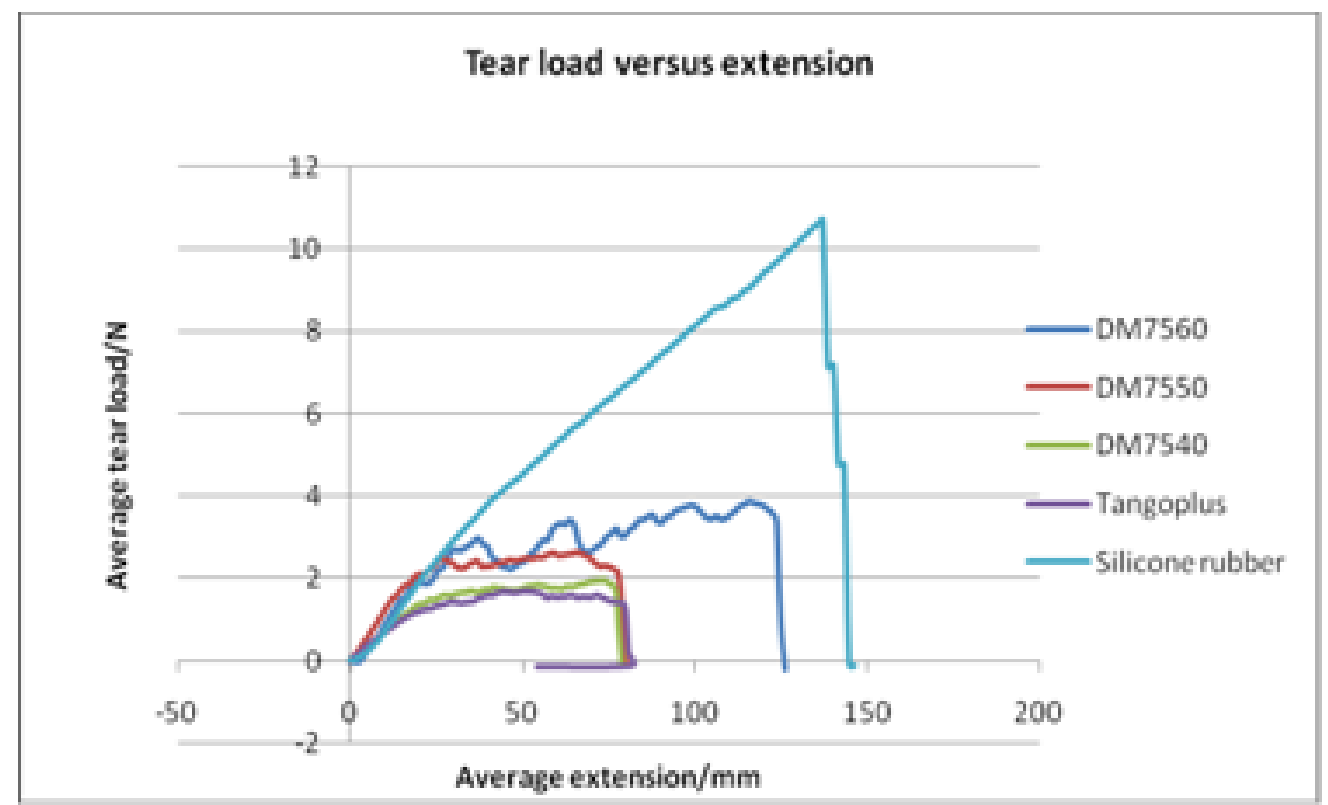

Figure 15: Tear load versus extension of Objet Connex materials and silicone rubber for maxillofacial prosthetics (Adapted from Eggbeer et al., 2012)

However, further research is required to explore these areas further, with opportunities to establish interventions to overcome these limitations. Since the underlying research focused on the 3D CAD processes to design splints for AM, performing mechanical tests on the prototypes was outside the remit of this investigation. The authors have performed research using Finite Element Analysis on a number of the proposed designs to enable comparison of the mechanical properties with traditional practice and this work will be published in due course. Structural analysis of homogeneous and heterogeneous splint builds will be performed by the authors to compare results with splints made with traditional fabrication methods and techniques. Concerns relating to UV exposure of photopolymer resin processes such as material jetting and SLA builds also need further exploration in an attempt to resolve breakdown of elastomer elements, as suggested by Eggbeer et al. (2012). 
Similar efforts should be performed on prevention of creep and discolouration of such materials and the AM processes used, particularly for Polyjet builds. Work is also being performed into the effects of material exposure to mechanical cleaning and everyday household chemicals, such as washing powders, liquids and other detergents, as this could potentially lead to suggestions into cleaning techniques for splints. As described in Section 4.1, the authors have begun preliminary testing of cleaning splints in a dishwasher, and as such will form further research to determine suitable variables for extending the life of a splint.

During Section 1 (Introduction), the authors identified that previous researchers had limitations relating to clinical validity for the use of upper extremity splinting. Similarly, the authors also recognise and acknowledge that significant future research is required with respect to their own documented work before conducting clinical trials in order to consider the efficacy of the approach for end use applications. This includes the exploration of analytical studies relating to all design work to consider material properties and structural integrity. The authors also acknowledge that further work must be explored into suitable data capture methods to support the digitised splinting approach for suggested improvements to fit and function. In addition, comments relating to surface finish and surface roughness were not quantified but preliminary in this case. Therefore, further research is required to quantify the surface roughness of AM splints, and the potential effect with the skin if worn by a patient, such as abrasion. Lastly, the current research is limited in terms of the usability of splints, since developments to date have not yet allowed for clinical trials. Further work will be required to assess the usability of different splint designs relative to a range of manual tasks (e.g. driving) which patients can compare and contrast against their previously prescribed splints made with traditional methods.

\section{Acknowledgements}

Many thanks to Dr. Candice Majewski of the University of Sheffield and Dr. Dominic Eggbeer and Sean Peel of the National Centre for Product Design \& Development Research, Cardiff for building the SLS and SL prototype splints respectively. Thanks to Nigel Bunt and Sarah Drage of HK Rapid Prototyping Ltd. for building the black and white Objet Connex splint, to Mark Tyrtania at LaserLines for the FDM splint, and to Phil Dixon, Loughborough University for assistance with the building of numerous Objet Connex prototypes. The research was carried out as part of a PhD research project at Loughborough University. 


\section{References}

AUSTIN, N.M., 2003a. Equipment and Materials. In: M. JACOBS and N.M. AUSTIN, eds, Splinting the Hand and Upper Extremity: Principles and Process. Baltimore: Lippincott Williams \& Wilkins, pp. 7387.

AUSTIN, N.M., 2003b. Process of Splinting. In: M. JACOBS and N.M. AUSTIN, eds, Splinting the Hand and Upper Extremity: Principles and Process. Baltimore: Lippincott Williams \& Wilkins, pp. 88-99. BIBB, R., 2006. Medical Modelling: The Application of Advanced Design and Development Techniques in Medicine. Cambridge: Woodhead Publishing.

BIBB, R., EGGBEER, D., EVANS, P., BOCCA, A. and SUGAR, A., 2009. Rapid manufacture of customfitting surgical guides. Rapid Prototyping Journal, 15(5), 346-354.

BINGHAM, G.A. and HAGUE, R.J.M., 2013. Efficient Three Dimensional Modelling of Additive Manufactured Textiles. Rapid Prototyping Journal, 19(4), 269-281.

BINGHAM, G.A., HAGUE, R.J.M., TUCK, C.J., LONG, A.C., CROOKSTON, J.J. and SHERBURN, M.N., 2007. Rapid Manufactured Textiles. International Journal of Computer Integrated Manufacturing, 20(1), 96-105.

BREUNINGER, J., 2010, Voronoi wrist splint [conversation] (personal communication, 26 November 2010).

CALLINAN, N.J. and MATHIOWETZ, V., 1996. Soft Versus Hard Resting Hand Splints in Rheumatoid Arthritis: Pain Relief, Preference and Compliance. The American Journal of Occupational Therapy, 50(5), 347-353.

CAMPBELL, R.I., 2006. Customer Input and Satisfaction. In: N. HOPKINSON, J.M. HAGUE and P.M. DICKENS, eds, Rapid Manufacturing: An Industrial Revolution for the Digital Age. Chichester: John Wiley \& Sons, Ltd., pp. 19-38.

COOK, D., GERVASI, V., RIZZA, R., KAMARA, S. and XUE-CHENG, L., 2010. Additive fabrication of custom pedorthoses for clubfoot correction. Rapid Prototyping Journal, 16(3), 189-193.

COPPARD, B., 2001. Anatomical and Biomechanical Principles of Splinting. In: B.M. COPPARD and H. LOHMAN, eds, Introduction to Splinting: A clinical-reasoning \& problem-solving approach. Second Edition. St Louis, MO.: Mosby, Inc., pp. 34-72.

COPPARD, B.M. and LYNN, P., 2001. Introduction to Splinting. In: B.M. COPPARD and H. LOHMAN, eds, Introduction to Splinting: A clinical reasoning \& problem-solving approach. Second Edition. St Louis, MO.: Mosby, Inc., pp. 1-33.

EGGBEER, D., 2008. The Computer Aided Design and Fabrication of Facial Prostheses, PhD Thesis. University of Wales

EGGBEER, D., BIBB, R., EVANS, P. and JI, L., 2012. Evaluation of direct and indirect additive manufacture of maxillofacial prostheses. Proceedings of the Institution of Mechanical Engineers, Part H: Journal of Engineering in Medicine, 226(9), 718-728.

EVILL, J., 2013. Cortex [online]. Available at: http://jakevilldesign.dunked.com/cortex [Accessed 01 August, 3013].

FAUSTINI, M.C., NEPTUNE, R.R., CRAWFORD, R.H. and STANHOPE, S.J., 2008. Manufacture of passive dynamic ankle-foot orthoses using selective laser-sintering. IEEE Transactions of Biomedical Engineering, 55(2), 784-790.

FRIED, S., 2007. Splint and or Method of Making Same. US Patent application 20070016323. FRIED, S., MICHAS, L. and HOWARD, J., 2004. Method of providing centralized splint production. US 20050015172.

GERVASI, V., COOK, D., RIZZA, R. and KAMARA, S., 2009. Fabrication of Custom Dynamic Pedorthoses for Clubfoot Correction via Additive-Based Technologies, Proceeding of the 20thAnnual International Solid Freeform Fabrication Symposium, University of Texas at Austin, pp. 652-661.

GIBSON, K.S., WOODBURN, J., PORTER, D. and TELFER, S., 2013. Functionally optimised orthoses for early rheumatoid arthritis foot disease: A study of mechanisms and patient experience. Arthritis Care \& Research, In Press. 
GRZESIAK, A., 2010. Fraunhofer Additive Manufacturing Alliance - Highlights, current RTD activities and strategic topics, FRAUNHOFER, ed. In: Proceedings of the Additive Manufacturing International Conference, July 08, 2010, Loughborough University, pp. 14.

HOLMES, S., 2012. Objet Printers get Colour Treatment [online]. Available at: http://develop3d.com/blog/2012/05/objet-printers-get-colour-treatment [Accessed June 18, 2012]. JACOBS, M., 2003. Splint Classification. In: M. JACOBS and N. AUSTIN, eds, Splinting the Hand and Upper Extremity: Principles and Process. Baltimore: Lippincott Williams \& Wilkins, pp. 2-18.

JACOBS, M. and AUSTIN, N., 2003. Splint Fabrication. In: M. JACOBS and N. AUSTIN, eds, Splinting the Hand and Upper Extremity: Principles and Process. Baltimore: Lippincott Williams \& Wilkins, pp. 98157.

JOHNSON, A., BINGHAM, G.A. and WIMPENNY, D.I., 2013. Additive manufactured textiles for highperformance stab resistant applications. Rapid Prototyping Journal, 19(3), 199-207.

LOHMAN, H., 2001. Wrist Immobilisation splints. In: B.M. COPPARD and H. LOHMAN, eds, Introduction to splinting: A clinical reasoning \& problem-solving approach. Second Edition. St Louis, MO.: Mosby, Inc., pp. 139-184.

LOHMAN, H., POOLE, S.E. and SULLIVAN, J.L., 2001. Clinical Reasoning for Splint Fabrication. In: B.M. COPPARD and H. LOHMAN, eds, Introduction to Splinting: A Clinical \& Problem-Solving Approach.

Second Edition. St Louis, MO.: Mosby, Inc., pp. 103-138.

MAVROIDIS, C., RANKY, R., SIVAK, M., PATRITTI, B., DIPISA, J., CADDLE, A., GILHOOLY, K., GOVONI, L., SIVAK, S., LANCIA, M., DRILLIO, R. and BONATO, P., 2011. Patient specific ankle-foot orthoses using rapid prototyping. Journal of NeuroEngineering and Rehabilitation, 8(1), 1.

MOORE, J.P. and WILLIAMS, C.B., 2012. Fatigue Characterisation of 3D Printed Elastomer Material, Proceedings of the Twenty Third Annual International Solid Freeform Fabrication Symposium - An

Additive Manufacturing Conference, August 6-8 2012, pp. 641-655.

OXMAN, N., 2012. Imaginary Beings @ Centre Pompidou [online]. Available at:

http://materialecology.blogspot.co.uk/2012/05/imaginary-beings-centre-pompidou.html [Accessed June 18, 2012].

OXMAN, N., 2011. Variable Property Rapid Prototyping. Virtual and Physical Prototyping, 6(1), 3-31. OXMAN, N., 2010. Material-based design computation, Ph.D. Thesis. Massachusetts Institute of Technology.

PAGNOTTA, A., KORNER-BITENSKY, N., MAZER, B., BARON, M. and WOOD-DAUPHINEE, S., 2005. Static wrist splint use in the performance of daily activities by individuals with rheumatoid arthritis. The Journal of rheumatology, 32(11), 2136-2143.

PALLARI, J.H.P., DALGARNO, J., MUNGUIA, J., MURARU, L., PEERAER, L., TELFER, S. and WOODBURN, J., 2010. Design and Additive Fabrication of Foot and Ankle-Foot Orthoses, D. BOURELL, ed. In: Proceedings of the Twenty First Annual International Solid FreeForm Fabrication Proceedings - An Additive Manufacturing Conference, August 9-11, pp. 834-845.

PALOUSEK, D., ROSICKY, J., KOUTNY, D., STOKLASEK, P. and NAVRAT, T., 2013. Pilot study of the wrist orthosis design process. Rapid Prototyping Journal, 20(1),.

PATERSON, A.M., 2013. Digitisation of the Splinting Process: Exploration and Evaluation of a Computer Aided Design approach to support Additive Manufacture. PhD ThesisEdition. Loughborough: Loughborough University.

PATERSON, A.M., BIBB, R.J. and CAMPBELL, R.I., 2012. Evaluation of a digitised splinting approach with multiple-material functionality using Additive Manufacturing technologies, D. BOURELL, R.H. CRAWFORD, C.C. SEEPERSAD, J.J. BEAMAN and H. MARCUS, eds. In: Proceedings of the 23rd Annual International Solid Freeform Fabrication Symposium, 6-8 August, University of Texas at Austin, pp. 656-672.

PATERSON, A.M., BIBB, R.J. and CAMPBELL, R.I., 2010. A review of existing anatomical data capture methods to support the mass customisation of wrist splints. Virtual and Physical Prototyping Journal, 5(4), 201-207. 
POPTECH, 2009. Neri Oxman: On Designing Form [online]. Available at: http://www.youtube.com/watch?v=tx|4QR0GDnU [Accessed April 17, 2010].

SMITH, S., 2011a. Micraclefeet and Objet Bring Innovation to Braces [online]. Available at: http://www.deskeng.com/articles/aabamt.htm [Accessed 07 August 2012, 2012].

SMITH, S., 2011b. Miraclefeet and Objet Bring Innovation to Braces [online]. Available at: http://www.deskeng.com/articles/aabamt.htm [Accessed May 25, 2011].

STRATASYS, 2013. Objet Digital Materials ${ }^{T M}$ Data Sheets [online PDF] [online]. Available at: http://www.stratasys.com/materials/polyiet/ /media/879CBF2F6582406C9C1B629F4F9E05D5.ashx [Accessed 04 April, 2013].

SUMMIT, S. and TRAUNER, K.B., 2010a. Custom braces, casts and devices having limited flexibility and methods for designing and fabricating. US 20100138193A1.

SUMMIT, S. and TRAUNER, K.B., 2010b. Modular custom braces, casts and devices and methods for designing and fabricating. US 20100268135A1.

VEEHOF, M.M., TAAL, E., WILLEMS, M.J. and VAN DE LAAR, M.A.F.J., 2008. Determinants of the use of wrist working splints in rheumatoid arthritis. Arthritis Care \& Research, 59(4), 531-536.

WOHLERS, T.T., 2012. Wohlers Report 2012: Additive Manufacturing and 3D Printing State of the Industry. Annual Worldwide Progress Report. Colorado: Wohlers Associates, Inc. 